\title{
Are lung adenocarcinoma mutations shaping the immune microenvironment?
}

\author{
Philippe Rochigneux ${ }^{1,2,3}$, Edward B. Garon ${ }^{3}$ \\ ${ }^{1}$ Medical Oncology Department, Paoli-Calmettes Institute, Marseille, France; ${ }^{2}$ Immunity \& Cancer Team, Inserm, U1068, Centre de Recherche \\ en Cancérologie de Marseille, Paoli-Calmettes Institute, Aix-Marseille University, Marseille, France; ${ }^{3}$ David Geffen School of Medicine at the \\ University of California, Los Angeles, CA, USA \\ Correspondence to: Dr. Edward B. Garon. UCLA Translational Oncology Research Laboratory, David Geffen School of Medicine at UCLA, 2825 \\ Santa Monica Blvd., Suite 200, Santa Monica, CA 90404, USA. Email: EGaron@mednet.ucla.edu. \\ Comment on: Biton J, Mansuet-Lupo A, Pécuchet N, et al. TP53, STK11 and EGFR Mutations Predict Tumor Immune Profile and the Response to \\ anti-PD-1 in Lung Adenocarcinoma. Clin Cancer Res 2018. doi: 10.1158/1078-0432.CCR-18-0163.
}

Submitted Jul 11, 2018. Accepted for publication Jul 18, 2018.

doi: $10.21037 /$ tcr.2018.07.17

View this article at: http://dx.doi.org/10.21037/tcr.2018.07.17

Recently, Biton et al. published in Clinical Cancer Research the article "TP53, STK11 and EGFR Mutations Predict Tumor Immune Profile and the Response to anti-PD-1 in Lung Adenocarcinoma" (1). This article points out the challenging question of the interplay between tumoral mutations in lung adenocarcinoma (localized and metastatic) and the composition of immune microenvironment.

Since the introduction of immune checkpoint blockade (ICB) in lung cancer, several attempts have been made to develop predictive biomarkers of anti-PD-1 response. With respect to biomarkers in the tumor, $\mathrm{PD}-\mathrm{L} 1$ expression in immunohistochemistry (IHC) (2) and tumoral mutation burden (TMB) (3) are the most advanced, with other candidates emerging [e.g., MMR deficiency (4) and JAK1/2 mutations (5)]. With respect to the immune microenvironment, derived neutrophils (leukocytes minus neutrophils) ratio (6), baseline monocytes (7) or invigoration of exhausted CD8 T cells (8) are associated with interesting preliminary data regarding anti-PD-1 response. However, data exploring the relation between tumoral characteristics and immune microenvironment are very scarce. And obviously, having an integrated vision of tumor and microenvironment together is of major importance when a therapeutic decision is needed for the patient.

Biton et al. studied both localized lung adenocarcinoma treated by surgery ( $\mathrm{n}=221$ and 24$)$ and advanced stage lung adenocarcinoma $(n=32)$ treated with the PD-1 inhibitor nivolumab. Using IHC and next generation sequencing of 7 oncogenes and tumor suppressive genes, they found a relation between 3 of them (TP53, STK11, EGFR) and the tumor immune profile (TIP): TP53 mutated tumors had higher stromal CD8 densities and PD-L1 expression; STK11 mutated tumors had more neutrophils, less dendritic cells (DC) and stromal CD8; EGFR mutated tumors had more DC and less macrophages, PDL1+ and stromal CD8. More specifically, TP53-Mut/STK11-EGFRWT presented a very interesting immune profile, with increased CD8 (both in tumor and stroma), mature DC and PD-L1 expression, suggesting a stronger immunogenicity. Gene expression analysis by NanoString system revealed that this tumor type (TP53-Mut/STK11-EGFR-WT) had 20 up-regulated genes involved in 3 main pathways: chemotaxis, cytotoxicity and peptide presentation by MHC-I. Patients with this tumor type (TP53-Mut/STK11EGFR-WT) treated with anti-PD-1 had an improved PFS compared to STK11-Mut or EGFR-Mut tumors [median progression-free survival (PFS): 8.5 vs. 4.6 and 1.4 months respectively], albeit in a small cohort of 32 patients. The authors also note similar results in a previously published cohort by Rizvi et al. (9) [log rank test in pooled cohorts: TP53-Mut vs. triple WT: $\mathrm{P}=0.002$; PT53-Mut vs. STK11(or)EGFR-Mut: $\mathrm{P}=0.003$ ].

This study has some limitations. First, the vast majority of the analyzed samples were localized adenocarcinoma, which have pathological differences compared to advanced adenocarcinoma that are currently benefiting from PD-1 
inhibitors (10). Secondly, the combination of the 2 cohorts (local and Rizvi et al.) grouped 2 different anti-PD-1 (nivolumab and pembrolizumab) because the number of metastatic patients treated locally was small. Thirdly, the reason for the selection of this particular 7 genes panel is not detailed and might be guided by the team's previous experience. Similarly, in the 3 genes combination, the selection of TP53 and STK11 was based on differences based on TIP class, but the reason for selection of EGFR is unclear and could have been justified. Finally, it would have been interesting to get additional information about rALK, MET and ROS status.

However, this study addresses important the question of the relationship between tumor mutated cells and their immune microenvironment, which have until now been mainly limited to cancer cells lines (11) or mouse models (12). Furthermore, the biological data presented are consistent among IHC, gene expression and flow cytometry, with TP53-Mut/STK11-EGFR-WT lung adenocarcinoma having a particular immune profile with a microenvironment increased in CD8, PDL1+ and mature DC. Interestingly, one of the only human study available in this area also described that TP53 mutated tumors have an increased expression of immune checkpoints and activated T-effector (13). As this tumor type particularly benefits from PD-1 inhibitors, this information can be greatly valuable, notably if the assessments of PD-L1 or TMB are not technically possible. The authors' data in EGFR mutated tumors, displaying less CD8 stromal, CD8 intratumoral and PD-L1 expression, also provide additional biological explanations of the poor efficacy of PD-1 inhibitors in this population $(14,15)$. Surprisingly, in $K R A S$ mutations, the results are not consistent with the previously cited study (13) as the authors did not find any immunological changes between wild type and mutated tumors. The mutations of KRAS and TP53 separately did not impact the survival of patients treated by anti PD-1, but this interpretation must be cautious due to the small number of patients in the cohort $(n=32)$. Also, there has been a great deal of interest in KRAS mutant, STK11 mutant NSCLC having limited benefit with PD-1 inhibition and TP53 mutant NSCLC being more sensitive (16), but this analysis did not support a clear role for KRAS mutations in this selection.

The positioning of the different gene alterations and TIPs compared with existing tumor biomarkers (PD-L1 and TMB) is complex and was probably not the goal of the authors. PD-L1 expression was assessed in the different immune profiles, and in TIP1 (enriched with CD8 cells),
PD-L1 expression was a pejorative prognostic factor in univariate analysis $(\mathrm{HR}=2.0395 \% \mathrm{CI}, 1.16-3.54, \mathrm{P}=0.013)$. However, the TIPs were analyzed in patients with localized tumor, which prevents the assessment of predictive value of TIP and PD-L1 expression together in a PD-1 inhibitor setting. Of note, in the Rizvi cohort, tumors TP53-Mut/ STK11-EGFR-WT had statistically more non-synonymous mutations and candidate neoantigens than the triple WT tumors. This data is consistent with the classical "guardian of the genome" function of $\mathrm{p} 53$. The article validated these previous results but also described a higher tumoral expression of PD-L1 in TP53-Mut tumors.

By describing the distinct combinations of tumoral mutational status and cross-referencing it with the quantification of major immune cell populations of the microenvironment, this study gives a broad image of the genetic/immune interplay. The 3 main mutations (TP53, $S T K 11, E G F R)$ are easily available in clinical routine and could be an interesting alternative to TMB, that have several disadvantages including cost, a multiplicity of tests analyzing different regions of the genome and the absence of clear cut-off. To validate these promising findings, a prospective assessment of the described biomarkers' combination TP53-Mut/STK11-EGFR-WT in an antiPD-1 trial would be of particular interest.

\section{Acknowledgments}

Funding: Dr. Garon was in part supported by National Institutes of Health (R01 CA208403).

\section{Footnote}

Provenance and Peer Review: This article was commissioned and reviewed by the the Section Editor Wei Xu (Division of Respiratory Disease, Department of Geriatrics, the First Affiliated Hospital of Nanjing Medical University, Nanjing, China).

Conflicts of Interest: Dr. Garon reports funds to his institution from AstraZeneca, Boehringer Ingelheim, Bristol Myers Squibb, Eli Lilly, Genentech, Mirati, Merck, and Novartis. Dr. Rochigneux has no conflicts of interest to declare.

Ethical Statement: The authors are accountable for all aspects of the work in ensuring that questions related to the accuracy or integrity of any part of the work are appropriately investigated and resolved. 
Open Access Statement: This is an Open Access article distributed in accordance with the Creative Commons Attribution-NonCommercial-NoDerivs 4.0 International License (CC BY-NC-ND 4.0), which permits the noncommercial replication and distribution of the article with the strict proviso that no changes or edits are made and the original work is properly cited (including links to both the formal publication through the relevant DOI and the license). See: https://creativecommons.org/licenses/by-nc-nd/4.0/.

\section{References}

1. Biton J, Mansuet-Lupo A, Pécuchet N, et al. TP53, STK11 and EGFR Mutations Predict Tumor Immune Profile and the Response to anti-PD-1 in Lung Adenocarcinoma. Clin Cancer Res 2018. doi: 10.1158/1078-0432.CCR-18-0163.

2. Garon EB, Rizvi NA, Hui R, et al. Pembrolizumab for the treatment of non-small-cell lung cancer. N Engl J Med 2015;372:2018-28.

3. Hellmann MD, Ciuleanu TE, Pluzanski A, et al. Nivolumab plus Ipilimumab in Lung Cancer with a High Tumor Mutational Burden. N Engl J Med 2018;378:2093-104.

4. Le DT, Uram JN, Wang H, et al. PD-1 Blockade in Tumors with Mismatch-Repair Deficiency. N Engl J Med 2015;372:2509-20.

5. Shin DS, Zaretsky JM, Escuin-Ordinas H, et al. Primary Resistance to PD-1 Blockade Mediated by JAK1/2 Mutations. Cancer Discov 2017;7:188-201.

6. Mezquita L, Auclin E, Ferrara R, et al. Association of the Lung Immune Prognostic Index With Immune Checkpoint Inhibitor Outcomes in Patients With Advanced Non-Small Cell Lung Cancer. JAMA Oncol 2018;4:351-7.

7. Krieg C, Nowicka M, Guglietta S, et al. High-dimensional single-cell analysis predicts response to anti-PD-1

Cite this article as: Rochigneux $\mathrm{P}$, Garon EB. Are lung adenocarcinoma mutations shaping the immune microenvironment? Transl Cancer Res 2018;7(Suppl 7):S740S742. doi: 10.21037/tcr.2018.07.17 immunotherapy. Nat Med 2018;24:144-53.

8. Huang AC, Postow MA, Orlowski RJ, et al. T-cell invigoration to tumour burden ratio associated with antiPD-1 response. Nature 2017;545:60-5.

9. Rizvi NA, Hellmann MD, Snyder A, et al. Cancer immunology. Mutational landscape determines sensitivity to PD-1 blockade in non-small cell lung cancer. Science 2015;348:124-8.

10. Vignot S, Besse B, André F, et al. Discrepancies between primary tumor and metastasis: a literature review on clinically established biomarkers. Crit Rev Oncol Hematol 2012;84:301-13.

11. Shatz M, Shats I, Menendez D, et al. p53 amplifies Tolllike receptor 5 response in human primary and cancer cells through interaction with multiple signal transduction pathways. Oncotarget 2015;6:16963-80.

12. Busch SE, Hanke ML, Kargl J, et al. Lung Cancer Subtypes Generate Unique Immune Responses. J Immunol 2016;197:4493-503.

13. Dong ZY, Zhong WZ, Zhang XC, et al. Potential Predictive Value of TP53 and KRAS Mutation Status for Response to PD-1 Blockade Immunotherapy in Lung Adenocarcinoma. Clin Cancer Res 2017;23:3012-24.

14. Lee CK, Man J, Lord S, et al. Checkpoint Inhibitors in Metastatic EGFR-Mutated Non-Small Cell Lung Cancer-A Meta-Analysis. J Thorac Oncol 2017;12:403-7.

15. Lisberg A, Cummings A, Goldman JW, et al. A Phase II Study of Pembrolizumab in EGFR-mutant, PD-L1+, Tyrosine Kinase Inhibitor (TKI) Naïve Patients with Advanced NSCLC. J Thorac Oncol 2018. doi: 10.1016/ j.jtho.2018.03.035.

16. Skoulidis F, Goldberg ME, Greenawalt DM, et al. STK11/LKB1 Mutations and PD-1 Inhibitor Resistance in KRAS-Mutant Lung Adenocarcinoma. Cancer Discov 2018;8:822-35. 